\title{
Factors that Strengthen and Weaken Self-Regulation in Peer Rejected and Accepted Pupils
}

\author{
Radana Novakova Kroutilova, Karla Hrbackova, Zuzana Hrncirikova \\ Department of Pedagogical Sciences, Faculty of Humanities, Tomas Bata University in Zlín, Czech Republic
}

\begin{abstract}
Peer rejection, which is accompanied by the experience of social pain, can have a serious impact on the ability to create new relationships in the future and can cause a negative effect on selfconcept. Research shows that peer rejected pupils fail to self-regulate their own behavior. These mechanisms may be undermined by peer rejection. This qualitative study explored which factors strengthen and weaken the self-regulation of behavior in peer rejected and accepted pupils. Twelve pupils (aged 12-14 years) were purposively sampled on the basis of the intensity of peer preferences in the class (strongly accepted and strongly rejected pupils). Unstructured face-toface interviews in combination with the problem situation analysis from video recording, the metaphorical associative cards, and a sociometric (peer nomination) method were conducted. Using the grounded theory and factoring methods, common factors have been found to affect the self-regulation of pupils' behavior in the classroom: own power or powerlessness; fear of being or not being accepted by peers, internal denial or manifestation of emotions and to stay with yourself or with others. The results suggest that the use of self-regulation behavior alters under different contextual influences and the nature of the peer interaction. Furthermore, the nature of the peer interaction is largely influenced by the relationship to oneself. The results indicate that it is important to understand the importance that pupils attribute to themselves and others and that facilitating factors should be used individually to reduce the effect of inhibiting factors to improve pupils's self-regulation.
\end{abstract}

Keywords: self-regulation; peer interaction; peer acceptance; peer-rejection; relationship to oneself. 